\title{
Red wine consumption increases antioxidant status and decreases oxidative stress in the circulation of both young and old humans Michelle Micallef ${ }^{\dagger 1}$, Louise Lexis ${ }^{\dagger 2}$ and Paul Lewandowski* ${ }^{*} 3$
}

\author{
Address: ${ }^{1}$ School of Biomedical Sciences, University of Newcastle, New South Wales, Australia, ${ }^{2}$ School of Biomedical Sciences, Victoria University, \\ Victoria, Australia and ${ }^{3}$ School of Medicine Deakin University, Victoria, Australia \\ Email: Michelle Micallef - michelle.micallef@studentmail.newcastle.edu.au; Louise Lexis - louise.lexis@vu.edu.au; \\ Paul Lewandowski* - paul.lewandowski@deakin.edu.au \\ * Corresponding author †Equal contributors
}

Published: 24 September 2007

Nutrition Journal 2007, 6:27 doi:10.1/86/1475-289/-6-27

This article is available from: http://www.nutritionj.com/content/6/I/27

(C) 2007 Micallef et al; licensee BioMed Central Ltd.

This is an Open Access article distributed under the terms of the Creative Commons Attribution License (http://creativecommons.org/licenses/by/2.0), which permits unrestricted use, distribution, and reproduction in any medium, provided the original work is properly cited.
Received: 19 December 2006

Accepted: 24 September 2007

\begin{abstract}
Background: Red wine contains a naturally rich source of antioxidants, which may protect the body from oxidative stress, a determinant of age-related disease. The current study set out to determine the in vivo effects of moderate red wine consumption on antioxidant status and oxidative stress in the circulation.

Methods: 20 young ( $18-30 \mathrm{yrs}$ ) and 20 older ( $\geq 50 \mathrm{yrs}$ ) volunteers were recruited. Each age group was randomly divided into treatment subjects who consumed $400 \mathrm{~mL} /$ day of red wine for two weeks, or control subjects who abstained from alcohol for two weeks, after which they crossed over into the other group. Blood samples were collected before and after red wine consumption and were used for analysis of whole blood glutathione (GSH), plasma malondialdehyde (MDA) and serum total antioxidant status.

Results: Results from this study show consumption of red wine induced significant increases in plasma total antioxidant status $(P<0.03)$, and significant decreases in plasma MDA $(P<0.00 \mathrm{I})$ and GSH $(P<0.004)$ in young and old subjects. The results show that the consumption of $400 \mathrm{~mL} /$ day of red wine for two weeks, significantly increases antioxidant status and decreases oxidative stress in the circulation
\end{abstract}

Conclusion: It may be implied from this data that red wine provides general oxidative protection and to lipid systems in circulation via the increase in antioxidant status.

\section{Background}

Efforts to define the role of nutrition in health have captured researcher's interest in antioxidants and their capacity to protect the body from damage induced by oxidative stress. Extensive research has demonstrated the protective properties of antioxidants, which scavenge reactive oxygen species (ROS) and their precursors, as well as up-regulate enzymes involved in the repair of cellular damage [1]. Red wine contains a rich source of a large number of antioxidants, namely the phenolic acids and polyphenols, which provide it with its protective redox potential $[2,3]$.

Epidemiological studies have shown that despite the high intake of saturated fatty acids within the diets of some populations, a reduced mortality rate from cardiovascular disease is attributed to the high consumption of red wine, independent of its alcohol content, the 'French Paradox' [4]. Studies also indicate that sub-populations already at a 
high risk of coronary heart disease (CHD) (i.e. elderly) may potentially experience a greater beneficial effect from moderate wine consumption [5]. Moderate consumption of red wine has also been shown to retard or slow the plasma clearance of high density lipoproteins (HDL), a negative risk factor for the development of cardio vascular disease (CVD). In doing so, a positive correlation between HDL particles and moderate red wine intake becomes evident [6]. Furthermore, the incubation of low density lipoproteins LDL) in varying concentrations of red and white wine showed a $50 \%$ decline in oxidation at concentrations of 0.04 and $0.7 \mathrm{mg} / \mathrm{ethanol} / \mathrm{mL}$ respectively, up to a concentration of $1.0 \mathrm{mg} / \mathrm{mL}$. These results indicate that red wine inhibits cell mediated LDL oxidation more efficiently then white wine and at much lower concentrations.

To investigate further, the relationship between red wine consumption and oxidative damage in humans has been studied by Greenrod and Fenech [7], in a series of in vitro and ex vivo study designs. They demonstrated a strong (> $70 \%$ ) reduction in $\mathrm{H} 2 \mathrm{O} 2$ induced genetic damage after 1hour post consumption of $300 \mathrm{~mL}$ of red wine. These findings are also supported by a similar study by Szeto and Benzie [8], showing that DNA damage was significantly reduced in a $\mathrm{H} 2 \mathrm{O} 2$ challenge, with treatment of caffeic acid, a polyphenol found in red wine.

Oxidative damage to a range of biomolecules is of particular interest to researchers. The tripeptide glutathione (GSH) functions as an antioxidant, which scavenges free radical species in circulation. GSH is oxidized as the enzyme glutathione peroxidase catalyzes the degradation of $\mathrm{H} 2 \mathrm{O} 2$ [9]. Increasing evidence demonstrates GSH plays an integral role in the protection against oxidative stress in the circulation due to its ability to facilitate the recycling of oxidized $\alpha$-tocopherol and ascorbic acid, two important antioxidants in the circulation and is widely used as a biomarker of circulating antioxidant levels [10]. Within plasma fatty acid residues of phospholipids and LDL, are extremely susceptible to oxidative damage by free radical intermediates resulting in oxidized fatty acids and peroxidation byproducts, such as conjugated diennes (CD) and malondialdehyde (MDA) derivatives [11]. MDA appears to be one of the most toxic and mutagenic aldehydes generated by lipid peroxidation of polyunsaturated fatty acids of cell membranes [12]. It is also a popular measurement used to quantify the effects of radical damage to cellular lipids [13].

A large body of evidence which indicates that free radical production can directly or indirectly play a major role in cellular processes implicated in atherosclerosis and CVD, [14]. Therefore the aim of this study were firstly to understand how moderate red wine consumption ( $400 \mathrm{ml} /$ day) for two weeks effected circulating lipids, antioxidant level and total antioxidant capacity in the circulation and secondly assess the differences in bioefficacy of red wine in young and older populations.

\section{Methods}

\section{Recruitment of volunteers}

This study protocol was approved by the Human Research Ethics Committee of Victoria University (HRETH.SET 15/ $05)$. Forty volunteers were selected based upon their responses to a general health questionnaire and after giving written informed consent. Those who were taking any anti-coagulant or anti-inflammatory medications or had a history of cardiovascular or liver disease were excluded. Two age groups were selected, these were 20 volunteers aged between 18-30 years old (young group) and 20 volunteers aged older then 50 years old (older group). Volunteers were randomly assigned to begin in the red wine or control group within their respective age group (Figure 1).

\section{Intervention design}

Prior to drinking the red wine or control period volunteers were asked to abstain from consuming any alcohol, grapes or grape products for one week. After this one week lead in subjects had three $10 \mathrm{~mL}$ tubes of fasting blood collected via venipuncture to determine baseline measures of MDA, GSH, and total antioxidant capacity and BMI $\left(\mathrm{kg} / \mathrm{m}^{2}\right)$ calculated, after which they began the red wine or control period. During the red wine period participants consumed $400 \mathrm{~mL}$ of red wine each day (Cabernet

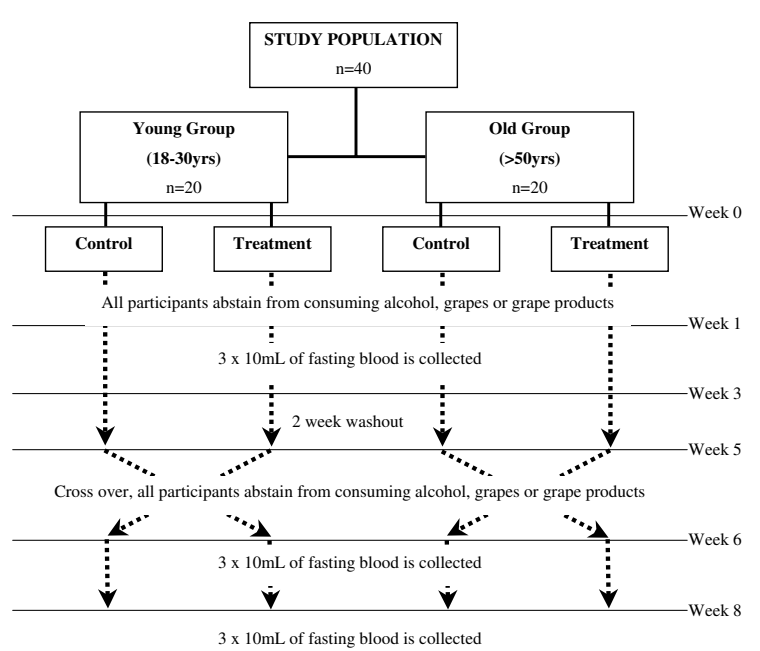

Figure I

Outline of study. Volunteers consumed $400 \mathrm{~mL} /$ day of red wine for two weeks or abstained from consuming alcohol, grapes or grape products for two weeks and crossed over into the other group after a two week washout. 
Sauvignon) over a period of two consecutive weeks and abstained from other alcohol, grapes or grape products. A placebo such as alcohol free wine was not used due to difficulties in matching the flavour and mouth feel of the red wine used. Instead a crossover design was used whereby after completing either the red wine or control period volunteers were given a two week washout period before crossing over into the other group. During the control period volunteers abstained from consuming any source of alcohol, grapes or grape products for two weeks. Three $10 \mathrm{~mL}$ tubes of fasting blood were again collected after the treatment or control phase (see Figure 1). Participants were also encouraged to maintain their usual diet and exercise habits throughout the entire study phase which was monitored by participants keeping a food and activity diary before and during the study. There were no specific instructions given to avoid foods containing large amounts of phenolic compounds, other than abstain from consuming any alcohol, grapes or grape products as previously described.

\section{Wine supplementation}

The red wine used throughout this study was a Cabernet Sauvignon, supplied as a cask wine to prevent the oxidation of the wine. This style was chosen since it is known to be palatable to most people and to the volunteers in the study. Participants consumed the wine at any time during the day, however, it was suggested that they do so at a time when they would normally consume alcohol (e.g. with an evening meal). Importantly, during the period of supplementation participants were asked to refrain from consuming any other sources of alcohol, grapes or grape products.

\section{Wine composition}

The concentrations of total anthocyanins, degree of anthocyanin ionisation, total phenolic compounds, red wine colour (density and hue) and two indices providing a measure of polymerisation of monomeric forms (Chemical age index \# 1 and \#2) were determined by spectrophotometric methods $[15,16]$. Determination of the concentration of free and bound sulphur dioxide in the wine was made using the method of Rankine and Pocock [17]. Alcohol content was provided by the wine producer. The composition of the wine used in this study was analysed can be seen in Table 1. All components of the wine used in this study, except for red wine colour - hue and free sulfur dioxide, were slightly higher than the red wine used in a study by Greenrod et al[18].

\section{Analyses of glutathione}

Glutathione was measured as it is an important antioxidant in the circulation using a commercially available colorimetric kit (Northwest Life Sciences) based on the method of Teitze [19] following the manufactures instruc-
Table I: Composition of wine used in study

\begin{tabular}{ll}
\hline Compounds & Amount \\
\hline $\begin{array}{l}\text { Total Anthocyanins } \\
\text { Degree of ionisation of } \\
\text { anthocyanins }\end{array}$ & $129 \mathrm{mg} / \mathrm{L}$ \\
$\begin{array}{l}\text { Degree of ionisation of } \\
\text { anthocyanins-abolishing effect of }\end{array}$ & $24.85 \%$ \\
sulphur dioxide & $31.56 \%$ \\
$\begin{array}{l}\text { Total phenolics } \\
\text { Total polyphenols }\end{array}$ & 40 a.u. \\
$\begin{array}{l}\text { Red wine colour - density } \\
\text { Red wine colour - hue }\end{array}$ & $1.558 \mathrm{~g} / \mathrm{L}$ \\
$\begin{array}{l}\text { Chemical age index \#I } \\
\text { Chemical age index \#2 }\end{array}$ & 0.4 a.u. \\
$\begin{array}{l}\text { Free sulfur dioxide, excluding } \\
\text { anthocyanin bound sulphur }\end{array}$ & 0.53 a.u. \\
dioxide & 0.26 a.u. \\
Alcohol & $1.04 \mathrm{mg} / \mathrm{L}$ \\
\hline
\end{tabular}

a Epicatechin equivalents.

a.u. Absorbance units.

tions. Blood was collected via venipuncture using EDTA coated tubes and stored at $4{ }^{\circ} \mathrm{C}$. Whole blood samples were then deproteinated mixing aliquots with $100 \mathrm{ul}$ of cold 5\% metaphosphoric acid followed by centrifugation at $1500 \times \mathrm{g}$ for $5 \mathrm{~min}$, the supernatant was then removed and stored at $-20^{\circ} \mathrm{C}$ awaiting further analysis. All samples were then assayed for reduced GSH as a batch. This involved mixing $50 \mu \mathrm{L}$ of calibrators or samples with 50 $\mu \mathrm{L}$ DTNB reagent and $50 \mu \mathrm{L}$ glutathione reductase reagent in the wells of microplate. This reaction mix was then incubated at ambient temperature for $3 \mathrm{~min}$ after which $50 \mu \mathrm{L}$ NADPH reagent was added to all wells and absorbance values read at $405 \mathrm{~nm}$ with data collected at $15 \mathrm{sec}$ intervals for $3 \mathrm{~min}$. Absorbance values were then plotted as a function of time for each calibrator and sample. A calibration curve was then constructed by plotting the $\Delta$ A405/min for each calibrator as a function of the GSH concentration and the equation for the calibration curve was then used to calculate the concentration of GSH in all samples.

\section{Analyses of malondialdehyde}

Plasma malondialdehyde was as a marker of lipid peroxidation using a commercially available colorimetric kit (Northwest Life Sciences) following the manufactures instructions. Blood was collected via venipuncture using EDTA coated tubes, stored at $4{ }^{\circ} \mathrm{C}$ and plasma separated within 2 hrs by centrifugation at $3000 \times \mathrm{g}$ for 10 minutes at room temperature. Plasma samples were then stored at $-20^{\circ} \mathrm{C}$ awaiting further analysis. All samples were then assayed for MDA as a batch. This involved mixing $250 \mu \mathrm{L}$ calibrator or sample with $10 \mu \mathrm{L}$ of Butylated hydroxytoluene reagent, $250 \mu \mathrm{L}$ Phosphoric acid reagent and $250 \mu \mathrm{L}$ 2 -Thiobarbituric acid reagent. This reaction mix was then incubated at $60^{\circ} \mathrm{C}$ for $60 \mathrm{~min}$ followed by centrifugation 
at $10000 \times \mathrm{g}$ for $3 \mathrm{~min}$. Absorbance of calibrators and samples was then read at $532 \mathrm{~nm}$ in a spectrophotometer (Biorad). Absorbance values for calibrators were then used to construct a calibration curve and the equation for calibration curve was then used to calculate the concentration of MDA in all samples.

\section{Analyses of total antioxidant status}

Serum total antioxidant status (TAS) was determined for a quantitative assessment of in vivo antioxidant status using a commercially available kit (Randox) based on the trolox equivalent antioxidant capacity method of Miller [20] following the manufactures instructions. Blood was collected via venipuncture using serum separator tubes, stored at $4^{\circ} \mathrm{C}$ and serum separated within 2 hrs. Serum samples were then stored at $-20^{\circ} \mathrm{C}$ awaiting further analysis. All samples were then assayed for TAS as a batch. This involved mixing $20 \mu \mathrm{L}$ calibrator (6-hydroxy-2,5,7,8tetramethylchroman-2-carboxylic acid $1.79 \mathrm{mmol} / \mathrm{L}$ ) or sample with $1 \mathrm{ml}$ of chromogen (metmyoglobin 6.1 $\mu \mathrm{mol} / \mathrm{L}$, ABTS $610 \mu \mathrm{mol} / \mathrm{L}$ ) and incubating at $37^{\circ} \mathrm{C}$ for 3 min. Initial absorbance was then read at $600 \mathrm{~nm}$ in a spectrophotometer (Biorad). After which $200 \mu \mathrm{L}$ of substrate (hydrogen peroxide $250 \mu \mathrm{mol} / \mathrm{L}$ ) was added to calibrator and sample and incubated at $37^{\circ} \mathrm{C}$ for $3 \mathrm{~min}$. Final absorbance was then read at $600 \mathrm{~nm}$. The change in absorbance value for samples relative to the change in absorbance of the calibrator was then to calculate the TAS in all samples. The total antioxidant status of the red wine (Cabernet Sauvignon) used in this study was also measured using the same assay.

\section{Analyses of serum glucose and plasma lipids}

Serum glucose was determined using a commercial glucose oxidase reagent and standard (Thermo Electron Corporation). This involved mixing $3 \mu \mathrm{L}$ of calibrator or sample with $450 \mu \mathrm{L}$ of glucose oxidase reagent and incubating at $37^{\circ} \mathrm{C}$ for $5 \mathrm{~min}$. Absorbance of calibrators and samples was then read at $500 \mathrm{~nm}$ in a spectrophotometer (Biorad). The absorbance value of samples relative to the absorbance of the calibrator was then to calculate the glucose level in all samples. Plasma triglycerides were determined using commercially available colorimetric kit (Thermo Electron Corporation). This involved mixing 6 $\mu \mathrm{L}$ of calibrator or sample with $600 \mu \mathrm{L}$ of triglyceride reagent and incubating at $37^{\circ} \mathrm{C}$ for $3 \mathrm{~min}$. Absorbance of calibrators and samples was then read at $500 \mathrm{~nm}$ in a spectrophotometer (Biorad). The absorbance value of samples relative to the absorbance of the calibrator was then to calculate the triglyceride level in all samples. Total cholesterol was determined using commercially available colorimetric kit (Thermo Electron Corporation). This involved mixing $6 \mu \mathrm{L}$ of calibrator or sample with $600 \mu \mathrm{L}$ of cholesterol reagent and incubating at $37^{\circ} \mathrm{C}$ for $3 \mathrm{~min}$. Absorbance of calibrators and samples was then read at
$500 \mathrm{~nm}$ in a spectrophotometer (Biorad). The absorbance value of samples relative to the absorbance of the calibrator was then to calculate the cholesterol level in all samples. HDL cholesterol was determined using commercially available colorimetric kit (Thermo Electron Corporation). This involved mixing $4 \mu \mathrm{L}$ of calibrator or sample with $300 \mu \mathrm{L}$ of HDL reagent 1 and incubating at $37^{\circ} \mathrm{C}$ for $5 \mathrm{~min}$. After which $100 \mu \mathrm{L}$ of HDL reagent 2 was added to calibrator and sample and incubated at $37^{\circ} \mathrm{C}$ for $3 \mathrm{~min}$. Absorbance of calibrators and samples was then read at $600 \mathrm{~nm}$ in a spectrophotometer (Biorad). The absorbance value of samples relative to the absorbance of the calibrator was then to calculate the triglyceride level in all samples. LDL cholesterol, a risk factor for cardiovascular disease, was calculated by subtracting HDL cholesterol values, a negative risk factor for cardiovascular disease, from total cholesterol.

\section{Statistical analysis}

Statistical analysis was performed using the SPSS statistical package (version 12.0, SPSS Inc.). All data were distributed normally and expressed as mean \pm standard error of the mean (SEM). Data from young and older individuals were analyzed using a three way ANOVA to determine the effect of wine consumption within the young or old group, any difference between young and old groups and any difference between pre samples with the young or old group. Due to the cross over design of the study any difference between are not included in the analysis $s$ the primary focus of the research was to determine the effect of red wine consumption. In all cases a P value of $<0.05$ was considered statistically significant.

\section{Results}

Whole blood glutathione was measured as it is an important circulating antioxidant. Before and after red wine consumption GSH levels were elevated in older volunteers compared to young volunteers $(\mathrm{P}<0.001$, Figure 2$)$. Despite this difference between young and old volunteers consumption of red wine had the same effect with both the young and old groups causing significant reductions in GSH levels after red wine consumption, young with wine $(P=0.004)$ and older with wine periods $(P<0.001$, Figure 2). No significant changes in GSH level were observed in young and older groups without red wine.

Plasma malondialdehyde was measured as a biomarker of lipid peroxidation. Before and after red wine consumption MDA levels were reduced in older volunteers compared to young volunteers $(P<0.05$, Figure 3$)$. Despite this difference between young and old volunteers consumption of red wine had the same effect within both the young and old group causing significant reductions in MDA levels after red wine consumption, young with wine $(P<0.001)$ and older with wine periods $(P<0.001$, Figure 


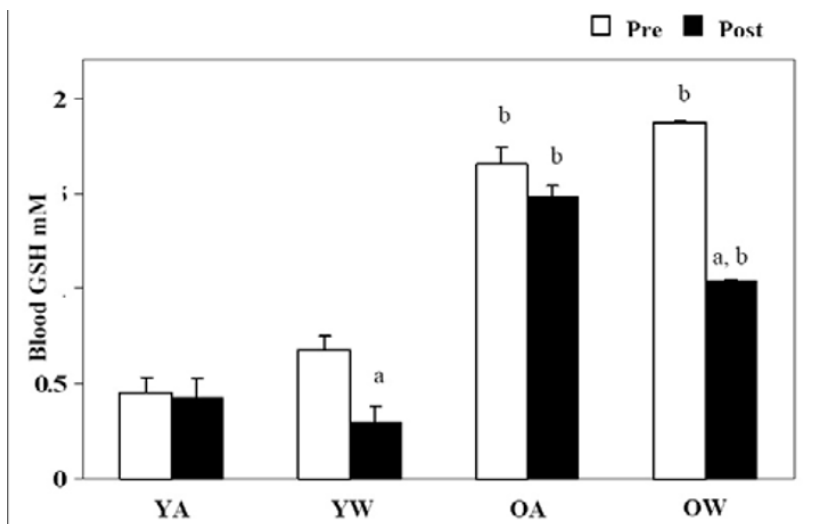

Figure 2

Blood glutathione. Effect of red wine consumption on blood GSH concentrations from young ( I 8-30 yrs) and older ( $\geq 50$ yrs) volunteers. Bars represent mean \pm SEM of blood GSH values pre and post two weeks of red wine consumption or abstinence. YA, young abstinence; $Y W$, young wine; OA, older abstinence; OW older wine. a indicates a significant change with wine $P<0.05$. $b$ indicates a significant difference between young and old, $P<0.05$.

3). No significant changes in MDA level were observed in young and older groups without red wine.

Serum total antioxidant status was calculated for samples from each study group. Before red wine consumption TAS levels were decreased in older volunteers compared to young volunteers $(\mathrm{P}<0.001$, Figure 4$)$. Despite this difference between young and old volunteers consumption of red wine had the same effect within both the young and old group demonstrating a significant increase in total antioxidant status after red wine consumption, young with wine $(P=0.026)$ and older with wine periods $(P=$ 0.01 , Figure 4$)$. These changes correspond to the changes

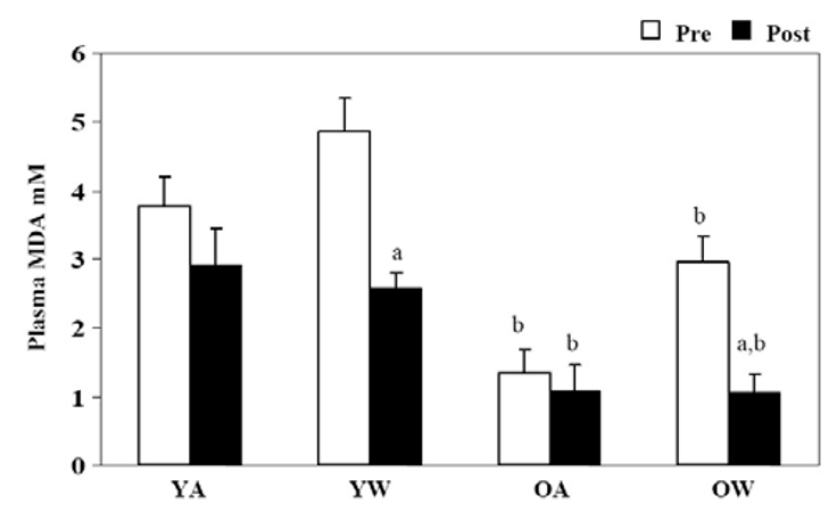

Figure 3

Plasma malondialdehyde. Effect of red wine consumption on plasma MDA concentrations from young (18-30 yrs) and older ( $\geq 50$ yrs) volunteers. Bars represent mean \pm SEM of plasma MDA values pre and post two weeks of red wine consumption or abstinence. YA, young abstinence; $Y W$, young wine; $O A$, older abstinence; $O W$ older wine. ${ }^{a}$ indicates a significant change with wine $P<0.05$. $b$ indicates a significant difference between young and old, $P<0.05$.

in GSH and MDA with red wine consumption for both young and older groups. The total antioxidant status of the red wine consumed by all treatment subjects in this study contained $1.53 \pm 0.027 \mathrm{mmol} / \mathrm{L}$ of antioxidant capacity (Figure 4 ).

There was no significant difference in both age (yrs) and BMI $(\mathrm{kg} / \mathrm{m} 2)$ between red wine and abstinence periods, for both young and older population groups (Table 2). Similarly there were no differences in serum glucose concentrations between pre and post samples for both young and older control and treatment groups (Table 2). Plasma

Table 2: Anthropometric and blood lipid measurements

\begin{tabular}{|c|c|c|c|c|c|}
\hline & & \multicolumn{2}{|c|}{ Young ( $18-30$ yrs) } & \multicolumn{2}{|c|}{ Older ( $\geq \mathbf{5 0}$ yrs $)$} \\
\hline & & Abstain & Red wine & Abstain & Red Wine \\
\hline Age (yrs) & & $21.7 \pm 0.3$ & $21.3 \pm 0.4$ & $58.9 \pm 2.3$ & $57.2 \pm 2.7$ \\
\hline BMI $\left(\mathrm{kg} / \mathrm{m}^{2}\right)$ & & $24.7 \pm 1.5$ & $23.6 \pm 1.2$ & $32.5 \pm 2.2$ & $28.3 \pm 2.2$ \\
\hline Cholesterol & Pre Sample & $4.3 \pm 0.2$ & $5.1 \pm 0.5$ & $5.9 \pm 0.3$ & $7.0 \pm 0.2$ \\
\hline (mmol/L) & Post Sample & $3.2 \pm 0.4$ & $5.1 \pm 0.1$ & $5.8 \pm 0.3$ & $7.2 \pm 0.2$ \\
\hline Triglycerides & Pre Sample & $\mathrm{I} .7 \pm 0 . \mathrm{I}$ & $1.96 \pm 0.2$ & $2.3 \pm 0.2$ & $2.1 \pm 0.1$ \\
\hline$(\mathrm{mmol} / \mathrm{L})$ & Post Sample & $1.8 \pm 0.1$ & $2.3 \pm 0.1$ & $2.5 \pm 0.1$ & $2.4 \pm 0.2$ \\
\hline HDL-Cholesterol & Pre Sample & $31.3 \pm 1.6$ & $33.6 \pm 2.4$ & $46.3 \pm 3.4$ & $42.1 \pm 2.4$ \\
\hline (mg/dL) & Post Sample & $37.1 \pm 2.6$ & $32.5 \pm 2.4$ & $44.3 \pm 1.5$ & $41.9 \pm 1.9$ \\
\hline LDL-Cholesterol & Pre Sample & $137.5 \pm 10.6$ & || $5 . \mid \pm 8.2$ & $194.8 \pm 10.4$ & $230.1 \pm 11.5$ \\
\hline (mg/dL) & Post Sample & $108.0 \pm 11.1$ & $131.9 \pm 8.8$ & $185.9 \pm 10.4$ & $225.5 \pm 12.8$ \\
\hline Glucose & Pre Sample & $5.8 \pm 0.3$ & $6.1 \pm 0.4$ & $6.6 \pm 0.5$ & $6.2 \pm 0.3$ \\
\hline (mmol/L) & Post Sample & $5.8 \pm 0.3$ & $6.1 \pm 0.5$ & $6.4 \pm 0.46$ & $6.5 \pm 0.5$ \\
\hline
\end{tabular}

Mean values for age and BMI of young (I8-30 yrs) and older ( $\geq 50$ yrs) subjects, together with serum glucose and plasma lipid profiles of pre and post samples for each study group. Values are mean \pm SEM. 


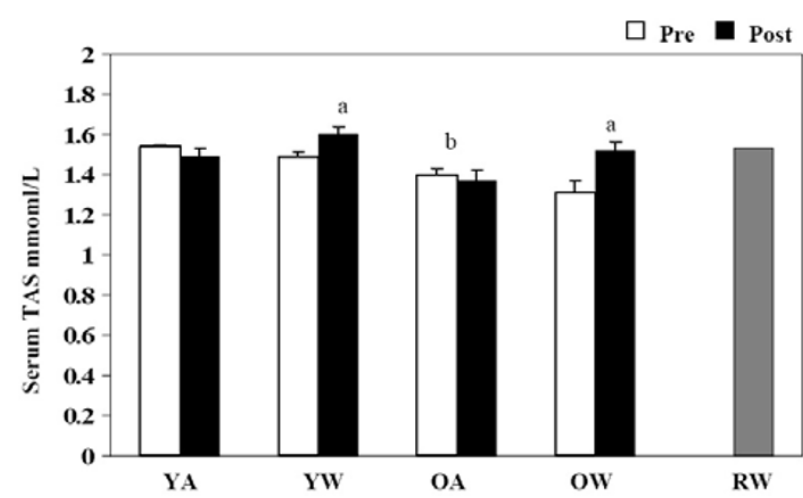

Figure 4

Serum total antioxidant status. Effect of red wine consumption on serum TAS from young ( $18-30 \mathrm{yrs}$ ) and older ( $\geq 50$ yrs) volunteers. Bars represent mean \pm SEM of serum TAS pre and post two weeks of red wine consumption or abstinence. YA, young abstinence; YW, young wine; OA, older abstinence; $\mathrm{OW}$ older wine; $\mathrm{RW}$ red wine a indicates a significant change with wine $P<0.05$. $b$ indicates a significant difference between young and old, $\mathrm{P}<0.05$.

lipid profiles for each study group were examined through the determination of plasma cholesterol, plasma triglycerides, plasma HDL-cholesterol and plasma LDL-cholesterol values. No statistical significance was found for any of the blood lipid profiles within each study group (Table 2).

\section{Discussion}

The results of these experiments show that moderate consumption of red wine significantly altered redox balance in the circulation of both young and old individuals. Red wine consumption increased serum antioxidant capacity and decreased concentrations of plasma malondialdehyde and whole blood glutathione in both age groups. In contrast, concentrations of malondialdehyde, glutathione, and total antioxidant capacity were significantly different between young and old control groups. Old subjects had greater concentrations of whole blood glutathione and lower concentrations of plasma malondialdehyde when compared to young subjects. Furthermore, total antioxidant capacity in serum was lower in the old group when compared to the young group. Red wine consumption did not significantly alter plasma lipid profiles and there were no significant differences between young and old individuals.

Oxidative stress is the consequence of an imbalance of oxidants and antioxidants. Studies show that a high consumption of antioxidants can decrease levels of oxidative stress and decrease the incidence of CVD [14]. In the current study TAS increased after red wine consumption these results strongly suggest that in the presence of red wine consumption, total antioxidant status has the ability to increase significantly. This increase was slightly more prominent in older individuals who increased their TAS $16 \%$ after consuming the red wine for two weeks compared to the younger individuals who increased $7 \%$, despite the fact that young individuals had higher resting concentrations of total antioxidant status. Our results are also shared in a study by Fernandez-Pachon and colleagues [21], who eluded that antioxidant values determined before wine intake were statistically different from those measured 30 minutes after consumption. Our study however, extends beyond Fernandez-Pachon's by advocating that the consistent consumption of wine may offer the ability to significantly enhance total antioxidant status over a longer period. This sustained elevation of TAS further confirms that the lower incidence of CVD in populations who consume red wine on a regular basis [14] is due to an increase in circulating oxidative protection. In addition it also suggests that a lifetime of red wine consumption is not needed to achieve a sustained increase in circulating oxidative protection, two weeks is long enough to boost TAS.

To further determine the extent of positive effects associated with and increase in TAS we measured MDA, as a biomarker of lipid peroxidation and found that there were significant reductions in MDA after red wine consumption for both young and older volunteers. This suggests that the consumption of red wine was also effective in protecting circulating lipid systems from oxidative damage in young and older volunteers. Whereas, young and older groups experienced no changes in MDA values without red wine. These results indicate that MDA as a marker of circulating lipid oxidation was significantly reduced, thereby representing a significant reduction in lipid peroxidation in participants who consumed the red wine. Our results are complimented by studies published by Fuhrman [22], which show that the propensity of lipids to undergo peroxidation was reduced by $20 \%$ in correspondence with the consumption of $400 \mathrm{~mL} /$ day of red wine for 2 weeks. Moreover, our results suggest that the antioxidant effect of dietary red wine on plasma lipid peroxidation could be related to the elevation of polyphenol concentration in plasma. Interestingly, the young group had higher levels of MDA compared with the older group prior to red wine consumption. This was an unexpected result, as theoretically it is believed that oxidative damage such as lipid peroxidation increases with age [23].

In addition to measuring TAS and MDA in the current study the levels of whole blood GSH were measured and it was found that red wine consumption decreased GSH in young and older volunteers. However, there were no significant reductions in GSH levels in the absence of red 
wine. This would suggest, that a reduction in GSH might be due to it being down regulated as a result of the increased level antioxidants derived from red wine eliminating additional reactive oxygen species. In addition, it would appear that the level of protection conferred was greatest amongst younger participants which demonstrated a 55\% reduction in GSH levels compared to older participants who had a $44 \%$ reduction in GSH, after both groups consumed red wine. It was expected and consequently demonstrated in this study, that the older population groups have a higher resting GSH content compared with their younger counterparts. This data may be interpreted with respect to the free-radical theory of ageing, in which endogenous oxidative damage occurs at higher frequencies with older age [24]. Data from Fenech et al [25] showed that the protective effects against DNA damage could not be readily explained by the phenolic content of the red wine, however, in a subsequent study by Greenrod et al [18] their data suggested that the nonalcoholic fraction of red wine protects DNA from oxidative damage, however, this effect is not solemnly explained by the antioxidant catechin.

No significant difference in age and BMI between young or old volunteers was observed before or after red wine consumption. This data indicates that potential changes in BMI had no influence on interpretation of the data. As the results of this present study indicate, no significant changes in plasma cholesterol, triglycerides, HDL-cholesterol, LDL-cholesterol and serum glucose were evidenced before and after red wine consumption (Table 2). Our data provides evidence that corresponds to research by Akcay and colleagues [26] whose findings suggest there is no significant difference in LDL blood levels with respect to the consumption of a cabernet sauvignon red wine.

From our data, relationships may be draw with respect to the consumption of red wine. We found that both young and older volunteers demonstrated significant decreases in GSH and MDA and this was proportional to the serum increases in total antioxidant status. This data signifies the relationship between a reduction in antioxidant levels and lipid oxidation and an increase in antioxidant status, with the consumption of $400 \mathrm{ml} /$ day of red wine for 2 weeks. This finding is important with respect to the long-term implications of red wine consumption. It suggests that the consistent consumption of red wine may confer prolonged oxidative protection, as opposed to conflicting research, which suggests plasma polyphenols only peak at 3 hours post consumption [21]. Our study controlled for these factors, where participants abstained from consuming any alternative alcoholic sources, grapes or grape products during the course of the study, as well as all blood samples were taken after 12-14 hrs fasting. Our findings shed further light on the nature of the beneficial effects of red wine consumption and gives supporting evidence for the recommendation that red wine provides protective effects for CVD. Also, drinking pattern and not just the total amount of red wine consumed is important in the association between intake and protection.

\section{Conclusion}

In conclusion red wine consumption decreases oxidative stress and enhances total antioxidant capacity in the circulation. This decrease in oxidative stress and increase in total antioxidant capacity in circulation is important as the opposite set of circumstances has been implicated in the pathogenesis of CVD. The results produced from this study suggest that the potent antioxidant properties provided by red wine and potential protection from developing CVD highlight the relationship between red wine consumption and health.

\section{Competing interests}

The author(s) declare that they have no competing interests.

\section{Authors' contributions}

MM carried out initial experimental procedures, data collection, preliminary data analysis and a 1 st draft of the manuscript. LL assisted in the drafting of the final manuscript interpretation of data. PL conceived, designed and coordinated the study, and carried out secondary experimental procedures and data collection to complete the crossover period of the study, together with drafting the manuscript.

\section{Acknowledgements}

We are grateful to the volunteers who participated in this study, who donated their time and commitment in the study.

\section{References}

I. Morton LW, Abu-Amsha Caccetta R, Puddey IB, Croft KD: Chemistry and biological effects of dietary phenolic compounds: relevance to cardiovascular disease. Clin Exp Pharmacol Physiol 2000, 27(3): I52-9.

2. Rice-Evans CA, Miller NJ, Bolwell PG, Bramley PM, Pridham JB: The relative antioxidant activities of plant-derived polyphenolic flavonoids. Free Radic Res 1995, 22(4):375-83.

3. German JB, Walzem RL: The health benefits of wine. Annu Rev Nutr 2000, 20:561-93.

4. Renaud S, de Lorgeril M: Wine, alcohol, platelets, and the French paradox for coronary heart disease. Lancet 1992, 339(8808): I523-6.

5. Gronbaek M: Factors influencing the relation between alcohol and mortality - with focus on wine. J Intern Med 2001, 250(4):29I-308.

6. Rifici VA, Stephan EM, Schneider SH, Khachadurian AK: Red wine inhibits the cell-mediated oxidation of LDL and HDL. J Am Coll Nutr 1999, I 8(2): I37-43.

7. Greenrod W, Fenech M: The principal phenolic and alcoholic components of wine protect human lymphocytes against hydrogen peroxide- and ionizing radiation-induced DNA damage in vitro. Mutagenesis 2003, 1 8(2): 1 19-26.

8. Szeto YT, Benzie IF: Effects of dietary antioxidants on human DNA ex vivo. Free Radic Res 2002, 36(I): I I3-8.

9. Tosukhowong P, Sangwatanaroj S, Jatuporn S, Prapunwattana P, Saengsiri A, Rattanapruks S, Srimahachota S, Udayachalerm W, Tang- 
kijvanich P: The correlation between markers of oxidative stress and risk factors of coronary artery disease in Thai patients. Clin Hemorheol Microcirc 2003, 29(3-4):32I-9.

10. Jefferies H, Coster J, Khalil A, Bot J, McCauley RD, Hall JC: Glutathione. ANZ J Surg 2003, 73(7):517-22.

II. Djuric Z, Potter DW, Taffe BG, Strasburg GM: Comparison of iron-catalyzed DNA and lipid oxidation. J Biochem Mol Toxicol 200I, I 5(2): I I4-9.

12. Lasheras C, Huerta JM, Gonzalez S, Brana AF, Patterson AM, Fernandez S: Independent and interactive association of blood antioxidants and oxidative damage in elderly people. Free Radic Res 2002, 36(8):875-82.

13. West IC: Radicals and oxidative stress in diabetes. Diabet Med 2000, I7(3): 17|-80.

14. Halliwell B, Gutteridge J, editors: Free radicals in biology and medicine. Oxford: Clarendon Press; 1989.

15. Somers T, Vérette E: Phenolic composition of natural wine types. In Modern Methods of Plant Analysis: Wine Analysis Edited by: Linskens H, Jackson J. Berlin: Springer-Verlag; 1988:219-57.

16. Somers T, Evans M: Spectral evaluation of young red wines: anthocyanin equilibria, total phenolics, free and molecular SO2 chemical age. J Sci Food Agric 1977, 28:279-87.

17. Rankine B, Pocock K: Alkalimetric determination of sulphur dioxide in wine. Aust Wine Brew Spirit Rev 1970, 88(8):40-4.

18. Greenrod W, Stockley CS, Burcham P, Abbey M, Fenech M: Moderate acute intake of de-alcoholized red wine, but not alcohol, is protective against radiation-induced DNA damage ex vivo - results of a comparative in vivo intervention study in younger men. Mutat Res 2005, 59 I (I-2):290-30I.

19. Tietze F: Enzymic method for quantitative determination of nanogram amounts of total and oxidized glutathione: applications to mammalian blood and other tissues. Anal Biochem 1969, 27(3):502-22.

20. Miller NJ, Rice-Evans C, Davies MJ: A new method for measuring antioxidant activity. Biochem Soc Trans 1993, 2 I (2):95S.

21. Fernandez-Pachon MS, Villano D, Troncoso AM, Garcia-Parrilla MC: Antioxidant capacity of plasma after red wine intake in human volunteers. J Agric Food Chem 2005, 53(I 2):5024-9.

22. Fuhrman B, Lavy A, Aviram M: Consumption of red wine with meals reduces the susceptibility of human plasma and lowdensity lipoprotein to lipid peroxidation. Am J Clin Nutr 1995, 6I(3):549-54.

23. Judge S, Jang YM, Smith A, Hagen T, Leeuwenburgh C: Age-associated increases in oxidative stress and antioxidant enzyme activities in cardiac interfibrillar mitochondria: implications for the mitochondrial theory of aging. Faseb J 2005, I9(3):4|9-2|.

24. Barnes DE, Lindahl T: Repair and genetic consequences of endogenous DNA base damage in mammalian cells. Annu Rev Genet 2004, 38:445-76.

25. Fenech M, Stockley C, Aitken C: Moderate wine consumption protects against hydrogen peroxide-induced DNA damage. Mutagenesis 1997, I 2(4):289-96.

26. Akcay Y, Yildirm H, Guvenc U, Sozmen E: The effects of consumption or organic and nonorganic red wine on low density lipoprotein oxidation and antioxidant capacity in humans. Nutrition Research 2004, 24(7):54I-54.
Publish with Biomed Central and every scientist can read your work free of charge

"BioMed Central will be the most significant development for disseminating the results of biomedical research in our lifetime. "

Sir Paul Nurse, Cancer Research UK

Your research papers will be:

- available free of charge to the entire biomedical community

- peer reviewed and published immediately upon acceptance

- cited in PubMed and archived on PubMed Central

- yours - you keep the copyright

Submit your manuscript here:

http://www.biomedcentral.com/info/publishing_adv.asp
BiolMedcentral 